\section{Radiación solar y planeamiento urbano: factores e interacciones en Bahía Blanca, Argentina}

\author{
María Eugenia Fernández \\ ORCID: https://orcid.org/0000-0002-6335-7774 \\ Correo electrónico: eugenia.fernandez@uns.edu.ar
}

\section{Jorge Osvaldo Gentili \\ ORCID: https://orcid.org/0000-0002-4787-4667 \\ Correo electrónico: jogentili@uns.edu.ar}

Filiación institucional: Departamento de Geografía y Turismo, Universidad Nacional del Sur; Consejo Nacional de Investigaciones Científicas y Técnicas (CONICET), Bahía Blanca, Argentina.

\section{Resumen}

El objetivo de este trabajo es analizar la distribución espaciotemporal de la radiación solar a escala urbana y su vinculación con elementos naturales y antropogénicos en Bahía Blanca (Argentina). Se generaron conocimientos relativos al clima solar urbano, insumo importante para la propuesta de medidas de desarrollo sostenible a escala local, lo que incluye el fortalecimiento de la infraestructura verde urbana. Se analizó la distribución temporal de la Radiación Solar Global (RSG) para el período 2000-2019 y su vinculación con las coberturas nubosas. Las nubes bajas y medias tienen mayor capacidad de reducción de la RSG y las nubes altas son las más transparentes. Se estudiaron descriptores morfológicos urbanos del microcentro que poseen influencia en la radiación: ancho y orientación de veredas, ancho de calles y altura de la edificación. La RSG en el microcentro es menor donde la edificación es más alta y compacta y donde los cañones urbanos son más profundos. La presencia de vegetación arbórea influye en los valores de RSG modelados. En las veredas con orientación noroeste y noreste, los valores de radiación son mayores. Se identificaron veredas aptas para la plantación de ejemplares arbóreos de acuerdo con el ancho de la acera, la orientación y los cañones urbanos.

\section{Palabras clave}

Heliofanía efectiva, infraestructura verde, morfología urbana, nubosidad, radiación solar

\section{Solar Radiation and urban planning: factors and interactions in Bahía Blanca, Argentina}

Financiamiento: El presente trabajo fue financiado por los proyectos "Radiación solar aplicada a la gestión local del cambio climático en Bahía Blanca" (24/ZG23) y "Geografía Física Aplicada al estudio de la interacción sociedad-naturaleza. Problemáticas a diversas escalas témporo-espaciales" (24/ G078), ambos con el subsidio de la Secretaría General de Ciencia y Tecnología, Universidad Nacional del Sur.

Agradecimientos: A las Lic. en Geografía María de los Ángeles Ortuño Cano, Belén Moretto y Marilina González por su colaboración en las campañas de relevamiento urbano. Al Servicio Meteorológico Nacional (SMN) por proveer los datos utilizados en el desarrollo de la presente investigación.

\begin{abstract}
The article analyzes the spatio-temporal distribution of solar radiation at urban scale and its relation with natural and anthropogenic elements in Bahía Blanca (Argentina). We provide specific knowledge related to the urban solar climate, an essential input for sustainable development local measures, including the improvement of urban green infrastructure. We analyzed the temporal distribution of global solar radiation (GSR) for 2000-2019 and its relationship with cloud cover, where low-level and mid-level clouds have the most significant capacity to reduce GSR, while high-level clouds are the most transparent. Urban morphological descriptors that affect downtown's solar access were analyzed, such as width and orientation of sidewalks, streets width, and buildings height. As findings, GSR is lower in areas with higher buildings, and the construction is compact, where the urban canyons are deeper. Likewise, vegetation in the central sector influences the GSR. Sidewalks with a northwest or northeast orientation have higher radiation values. Suitable sidewalks for planting trees were identified according to sidewalk width and orientation and the depth of the urban canyons.
\end{abstract}

\section{Keywords}

Cloud cover, green infrastructure, solar radiation, sunshine hours, urban morphology 


\section{Introducción}

El mundo se vuelve cada día más urbano. Ciertas estimaciones sugieren que para el año 2050, dos tercios de la humanidad vivirá en ciudades (Kamiya, \& Zhang, 2017; United Nations, 2015). Con la urbanización aumentará la frecuencia, intensidad y complejidad de los problemas ambientales. Por lo tanto, el planteamiento y aplicación de políticas públicas que generen ciudades sostenibles, confortables y resilientes constituye uno de los más grandes desafíos de este siglo.

En 2015 la Asamblea General de Naciones Unidas aprobó la Agenda 2030 para el Desarrollo Sostenible que incluye 17 Objetivos de Desarrollo Sostenible, entre ellos: garantizar el acceso a una energía asequible, segura, sostenible y moderna (objetivo 7); lograr que las ciudades sean más inclusivas, seguras, resilientes y sostenibles (objetivo 11); y adoptar medidas urgentes para combatir el cambio climático y sus efectos (objetivo 14) (Dugarova, \& Gülasan, 2017; United Nations General Assembly, 2015). En esta línea de acción, adquieren relevancia medidas que mitiguen el cambio climático y sus efectos, garanticen la sustentabilidad de los recursos renovables, la reducción del uso de los recursos no renovables, la prevención de la contaminación, la inversión en infraestructura ecológica y la resiliencia ante los desastres (European Enviroment Agency [EEA], 2015).

En este contexto, analizar la dimensión solar como herramienta para el desarrollo urbano sostenible posee cada día mayor y especial interés. El análisis holístico e integral de la radiación solar en entornos urbanos implica identificar dimensiones o aspectos desde donde abordar su estudio en relación con el hombre, su entorno físico-natural y sus actividades, organizados en tres categorías (Fernández, 2020). El sol puede ser visto como un recurso - sea desde un punto de vista de sostenibilidad y optimización energética-, de beneficio para la salud o como elemento de disfrute. El contacto directo con este produce sensación de bienestar, es necesario en la síntesis de vitamina $\mathrm{D}$ en el organismo y mejora afecciones dermatológicas (Gray Lovio et al., 2014). Por otro lado, la disponibilidad de luz solar directa en edificios privados y áreas públicas es un derecho (Fernández, 2020; M.E. Fernández y J.O. Gentili, comunicación personal, 31 de julio de 2021). Sin embargo, la exposición a la radiación solar puede suponer un peligro para el hombre y su salud. Durante el verano, la sobrexposición puede generar situaciones de disconfort térmico (Hwang et al., 2011; Kurazumi et al., 2013; Li \& Ratti, 2018; Zhao et al., 2013), golpe de calor o exacerbación de problemas respiratorios preexistentes, entre otras dolencias (Gasparrini et al., 2015; Gronlund et al., 2016; Klinenberg, 2015; Lee et al., 2018). Dichas dimensiones contemplan los usos del sol que deben ser tenidos en cuenta y garantizados en el entorno urbano: el individual (acceso directo de la luz diurna y a la iluminación natural de los edificios), el técnico (conversión de la radiación solar en distintas formas de energía) y el social (asoleo directo de áreas públicas) (Curreli, 2017; Treberspurg, 2008).

Debido a su constante crecimiento y a la dinámica de su desarrollo, Bahía Blanca no escapa a las problemáticas ambientales urbanas más comunes. El proceso de urbanización y la reducción de la biomasa en el interior de la ciudad produce cambios en las propiedades atmosféricas locales (Capelli de Steffens et al., 1989, 2005; Ferrelli, 2016) ) y en los flujos que integran el balance energético (Fernández et al., 2021) a escala urbana. La edificación vertical define patrones de iluminación y sombra que afectan el acceso al sol y, por lo tanto, posee consecuencias sobre el confort de los habitantes (Capelli de Steffens et al., 2003; Fernández et al., 2017). Por otro lado, la expansión urbana hacia la periferia favorece el uso del vehículo privado como alternativa a la demanda de movilidad (Schroeder y Garriz, 2009), afectando la calidad del aire en el área urbana. Además, la expansión del parque automotor y la frecuente construcción de edificios que no cuentan con cocheras hace que las playas de estacionamiento hayan aumentado su presencia en el ámbito urbano. Esta tipología de uso de suelo urbano representativa del microcentro de Bahía Blanca es cada vez más frecuente y repercute negativamente en el ambiente urbano, colabora con la Isla de Calor Urbana (ICU) y con la disminución de la calidad del aire en las zonas centrales (Gentili et al., 2020). Es evidente la necesidad de proponer y aplicar medidas orientadas a desarrollar y 
mantener una estructura urbana que requiera un menor uso del suelo y de la energía, contribuya al cuidado del ambiente y mejore las condiciones de vida y la salud de la población. En este contexto, una de las posibles líneas de abordaje es analizar la dimensión solar como herramienta de sostenibilidad en la ciudad. Ante esto, surgen las siguientes preguntas de investigación:

- ¿Cuál es la distribución espaciotemporal de la radiación solar en Bahía Blanca?

- ¿Qué factores naturales y antropogénicos definen dicha distribución y cómo lo hacen?

- ¿Qué medidas urbanísticas sostenibles pueden plantearse a partir de la generación de este conocimiento?

A fin de dar respuesta a estas preguntas, la hipótesis que guía la presente investigación es: la distribución espaciotemporal de la radiación solar en la ciudad se ve determinada por factores naturales y antropogénicos, y su conocimiento es un insumo de interés para generar propuestas urbanísticas sostenibles.

En esta línea, el objetivo de este trabajo es analizar la distribución espaciotemporal de la radiación solar a escala urbana y su vinculación con elementos naturales y antropogénicos en Bahía Blanca (Argentina). Para ello se estudia la vinculación de la variable con coberturas nubosas y la influencia de la estructura urbana en la radiación que alcanza la superficie. Este trabajo genera conocimientos relativos al clima solar a escala urbana, que constituye un insumo importante debido a su contribución para el planteamiento de medidas de desarrollo sostenible, lo que incluye el fortalecimiento de la infraestructura verde en las zonas centrales. La difusión de estrategias holísticas que contemplen todas las dimensiones y usos del sol en la estructura urbana permiten la consolidación de ciudades sostenibles, resilientes, con menor uso de energía y mejores condiciones de vida para la población.

\section{Marco teórico}

La radiación solar que alcanza cierto sector de la superficie terrestre se ve determinada por la altura solar, la duración del día, la topografía del terreno y el efecto de la atmósfera. La atmósfera terrestre constituye un constante filtro para la radiación. La radiación incidente sufre tres procesos al atravesar la atmósfera: absorción, difusión y reflexión (Abal, 2012; Oke et al., 2017; Patton et al., 1978; Sengupta et al., 2017). La dinámica de los sistemas de acción y las coberturas nubosas asociadas son las principales causas de la variabilidad espaciotemporal de la radiación solar (Matuszko, 2012; Weber, 1994). Para Bahía Blanca esto fue documentado en el análisis efectuado por Fernández, Gentili et al. (2018). La interacción de la radiación solar y sus componentes con la dinámica de los centros de acción es de interés a la hora de determinar el potencial energético renovable de un sitio en particular, por lo que ha sido estudiada por numerosos investigadores alrededor del mundo. Por ejemplo, Jerez y Trigo (2013) afirmaron que la variabilidad de la heliofanía efectiva en la península ibérica es mayor en la temporada fría debido en parte a la influencia de los patrones de circulación a gran escala. Pozo-Vázquez et al. (2004) obtuvieron resultados similares del análisis de la influencia de la North Atlantic Oscillation (NAO) en el comportamiento de la radiación solar en la región europea del Atlántico Norte. Según Tomson y Hansen (2010), debido a los ciclones atlánticos predominantes y frecuentes, la radiación solar tiene una naturaleza alterna en el norte de Europa. Por su parte, Urban et al. (2017) especificaron que la proporción limitada de ciclones occidentales en Polonia causa un bajo grado de nubosidad y valores máximos de heliofanía efectiva. A su vez, la reducción en el promedio de heliofanía efectiva es originada por la dominancia ciclónica occidental y el mayor grado de nubosidad. En el suroeste bonaerense (Argentina) y en la ciudad de Bahía Blanca, Fernández, Gentili et al. (2018) y Fernández (2020) verificaron que la dinámica radiactiva se ve en gran parte determinada por el pasaje de anticiclones migratorios en el territorio argentino y por las condiciones sinópticas asociadas, así como con el pasaje de frentes fríos. Asimismo, estudios relativos a la influencia de la nubosidad en la radiación solar de la ciudad documentaron que, en término medio, entre un día despejado y un día con cielo cubierto hay un $35 \%$ de diferencia en la radiación solar global recibida (Fernández et al., 2015) y una diferencia de cinco a siete horas en la heliofanía efectiva (Fernández, Gentili et al., 2018). 
Las complejas interacciones de la radiación solar con la atmósfera terrestre resultan en el abordaje teórico de sus tres componentes. La radiación directa es aquella que se recibe directamente del Sol, sin sufrir ninguna dispersión atmosférica. La radiación difusa es la que la superficie recibe del sol luego de ser desviada por las partículas discretas presentes en la atmósfera. La radiación solar global (RSG) es la suma geométrica de las radiaciones directa y difusa que se reciben sobre una superficie (Habte et al., 2017; Hermosillo Villalobos et al., 1995). La heliofanía efectiva se refiere a la suma de los períodos de tiempo en que la radiación solar directa excede los $120 \mathrm{~W} / \mathrm{m}^{2}$. El instrumental más utilizado para su registro por el Servicio Meteorológico Nacional es el heliofanógrafo tipo Campbell-Stokes. El registro se obtiene sobre una faja de papel que es quemada por los rayos del sol refractados en la esfera. Todo fenómeno meteorológico (litometeoros, nubes o nieblas) que intercepte la radiación solar directa quedará registrado en la faja al disminuir la intensidad del trazo, llegando, a veces, a no producirse registro alguno (World Meteorological Organization [WMO], 2017).

En las ciudades, los patrones de solanas y umbrías poseen una dinámica distintiva debido a las obstrucciones de edificios y árboles. Las variables morfológicas de las configuraciones urbano-edilicias limitan el acceso solar debajo del nivel de los edificios y crean sombras en los ambientes en los que los peatones circulan (Arboit, 2013; Arboit y Betman, 2014, 2017; Basso et al., 2013; Oke et al., 2017). Entre los principales descriptores morfológicos urbanos relacionados con las solanas y umbrías urbanas se encuentran la orientación de la trama urbana, el ancho de las calles y la altura de los edificios. La orientación de la trama urbana determina qué sectores estarán expuestos a los rayos directos del sol en una u otra época del año. La altura de los edificios define la proyección de su sombra sobre las fachadas de otros edificios o sectores públicos de la ciudad. El ancho de las calles dará cuenta de la proximidad física de las edificaciones, lo que reduce la penetración de la radiación solar (Curreli, 2017; Curreli et al., 2016). Una de las unidades elementales de la morfología urbana son los cañones urbanos, que se refieren al espacio formado por las paredes de los edificios y la calle entre ellos (Oke et al., 2017). La relación de aspecto (aspect ratio) es una medida ampliamente utilizada para conocer la geometría de los cañones urbanos, que define la relación entre el alto de los edificios (H) y el ancho de las calles (W). Este factor constituye un elemento fundamental en el análisis de la radiación solar y el balance energético a escala urbana (Carrasco Aldunate, 2010; Curreli, 2017; Rodríguez Algeciras, 2013; Shishegar, 2013).

El fortalecimiento de la infraestructura verde en la ciudad se propone como una medida fundamental para el confort, la salud y la protección frente a la exposición solar. La infraestructura verde incluye plantación de árboles, techos y paredes verdes (Arabi et al., 2015; Gill et al., 2007; Norton et al., 2015; Reyes, 2020). La plantación de especies arbóreas en los centros urbanos potencia los procesos de reflexión de radiación y evapotranspiración y contribuye a la disminución de la ICU, aportando sombra e incrementando el confort térmico de los habitantes (Bajsanski et al., 2016; Blanchard, \& Tanenbaum, 2003; Declet-Barreto et al., 2016; Gill et al., 2007; Goodrich, 2016; Knight et al., 2016; Onishi et al., 2010; Scott et al., 1999; Wong, \& Yu, 2005). Numerosos estudios han probado el efecto refrigerante de los árboles y el césped en el clima urbano (Arabi et al., 2015; Gill et al., 2007; Middel et al., 2015; Norton et al., 2015; Shahidan et al., 2012; Vásquez, 2016). Por ejemplo, Middel et al. (2015) estudiaron el efecto de la vegetación urbana sobre la temperatura en Phoenix (Arizona, USA). Los autores afirmaron que un aumento en la cobertura de la copa de los árboles del $10 \%$ actual a un $25 \%$ objetivo resultaría en un beneficio de enfriamiento diurno promedio de hasta $2,0^{\circ} \mathrm{C}$. Estudios similares llevados a cabo en Australia mostraron que, con el incremento de un $10 \%$ en la cobertura vegetal urbana, la temperatura diurna podría reducirse en $1{ }^{\circ} \mathrm{C}$ (Coutts, \& Harris, 2013). Por su parte, Onishi et al. (2010) documentaron una disminución de hasta $7,26{ }^{\circ} \mathrm{C}$ en verano al aumentar la presencia de vegetación en playas de estacionamiento. Adicionalmente, la vegetación tiene la capacidad de reducir los contaminantes a nivel troposférico, como el ozono, los óxidos de nitrógeno y el material particulado atmosférico (Acero Alejandro, 2010; Knight et al., 2016; Rowe, 2018). Al respecto, Rowe 
(2018) discutió la mitigación de la polución atmosférica producto de la instalación de techos verdes, Lazzari et al. (2018) estudiaron el efecto de la plantación de especies en cañones urbanos en la calidad del aire y Perini y Roccotiello (2018) analizaron el efecto de las paredes verdes en la concentración de material particulado atmosférico. En este sentido, deben evaluarse medidas para fortalecer la infraestructura verde de la ciudad, teniendo en cuenta su morfología y relación con la distribución diaria y estacional de la insolación en el ambiente urbano.

\section{Metodología}

\section{Área de estudio}

Bahía Blanca ( $38^{\circ} 44^{\prime}$ 'S y $\left.62^{\circ} 16^{\prime} \mathrm{O}\right)$ está ubicada al sur de la provincia de Buenos Aires, Argentina (Figura 1a). Constituye la ciudad cabecera del partido homónimo, que cuenta con 301.572 habitantes (Instituto Nacional de Estadísticas y Censos de la República Argentina [INDEC], 2010).

La alternancia de las masas de aire a nivel regional determina para Bahía Blanca un clima de transición entre el cálido y húmedo del este de la provincia de Buenos Aires y el frío y seco de la Patagonia. La circulación atmosférica regional imprime para la ciudad características continentales, a pesar de su posición costera (Capelli de Steffens et al., 2005). Bahía Blanca tiene una temperatura media anual de $15,5^{\circ} \mathrm{C}$, con una estacionalidad térmica marcada: $22,3^{\circ} \mathrm{C}$ la media estival y $9,5^{\circ} \mathrm{C}$ la media invernal. Las precipitaciones poseen un valor medio anual de 644,6 $\mathrm{mm}$. El verano es la estación más lluviosa en la ciudad, con un valor medio de 206,2 mm (Ferrelli, 2016; Zapperi, 2012). Esta se caracteriza por una gran variabilidad interanual de las precipitaciones. Las décadas 1960-1970 y 2000-2010 fueron las más secas de los últimos sesenta años (Ferrelli, 2016). Respecto del viento, sus direcciones preponderantes son norte y noroeste, con velocidades mayores durante el verano. Bahía Blanca registra uno de los mayores valores medios de velocidad de viento de la región (Campo de Ferreras et al., 2004).
Desde un punto de vista topográfico (Figura 3e), Bahía Blanca está emplazada, en su mayor parte, en dos niveles altitudinales, cada uno de ellos de escasa pendiente (entre $1 \%$ y $2 \%)$. El nivel más alto posee alturas superiores a los 80 m s.n.m., mientras que el más bajo cuenta con alturas entre el nivel del mar y los 20 o 30 m s.n.m (Figura 3e). La vinculación entre ambos niveles es mediante un talud de desarrollo aproximadamente latitudinal con gradientes que oscilan entre el $2 \%$ y el $8 \%$ (Gentili et al., 2020).

En la región donde se emplaza la ciudad convergen tres provincias fitogeográficas: la del espinal, la del monte y la pampeana (Cabrera, 1976). Bajo las condiciones naturales del sitio no se desarrollan grandes masas de vegetación arbórea. Por ende, la introducción de especies exóticas es una práctica común en las aceras de los barrios (arbolado de alineación) y en los espacios verdes (Benedetti et al., 2013; Benedetti y Campo de Ferreras, 2007) (Figura 1a). Los espacios verdes más valorados son la plaza Rivadavia, el parque Independencia, el parque de Mayo y el paseo de las Esculturas (Ercolani, 2005; Pinassi, 2016; Rosake y Ercolani, 2012).

La ciudad se ha consolidado como un centro de importancia regional, proveedor de bienes y servicios de la zona aledaña. Constituye un destacado nodo de transportes y comunicaciones con una amplia infraestructura terrestre, marítima y aeroportuaria (Lorda, 2008). Bahía Blanca ha sido protagonista de una expansión notable, materializada en superficie con la extensión desde el centro a la periferia y en altura. La edificación en altura comenzó a mediados del siglo XX en la zona central y en los años subsiguientes rebasó los límites del microcentro y macrocentro (Bróndolo et al., 1994; Fittipaldi et al., 2018; Formiga y Marenco, 2000). Es en el microcentro (Figura 1b) donde se concentran las actividades comerciales y de servicios de la ciudad y la mayor densidad de edificios en altura (Figura 1c). Debido a su constante crecimiento y a la dinámica de su desarrollo, la forma y función de Bahía Blanca posee consecuencias directas en el ambiente y en la calidad de vida de los habitantes. El proceso de urbanización y la reducción de la biomasa en el interior de la ciudad produce cambios en las propiedades atmosféricas locales y define la partición 
Figura 1

Área de estudio, teoría,métodos y técnicas

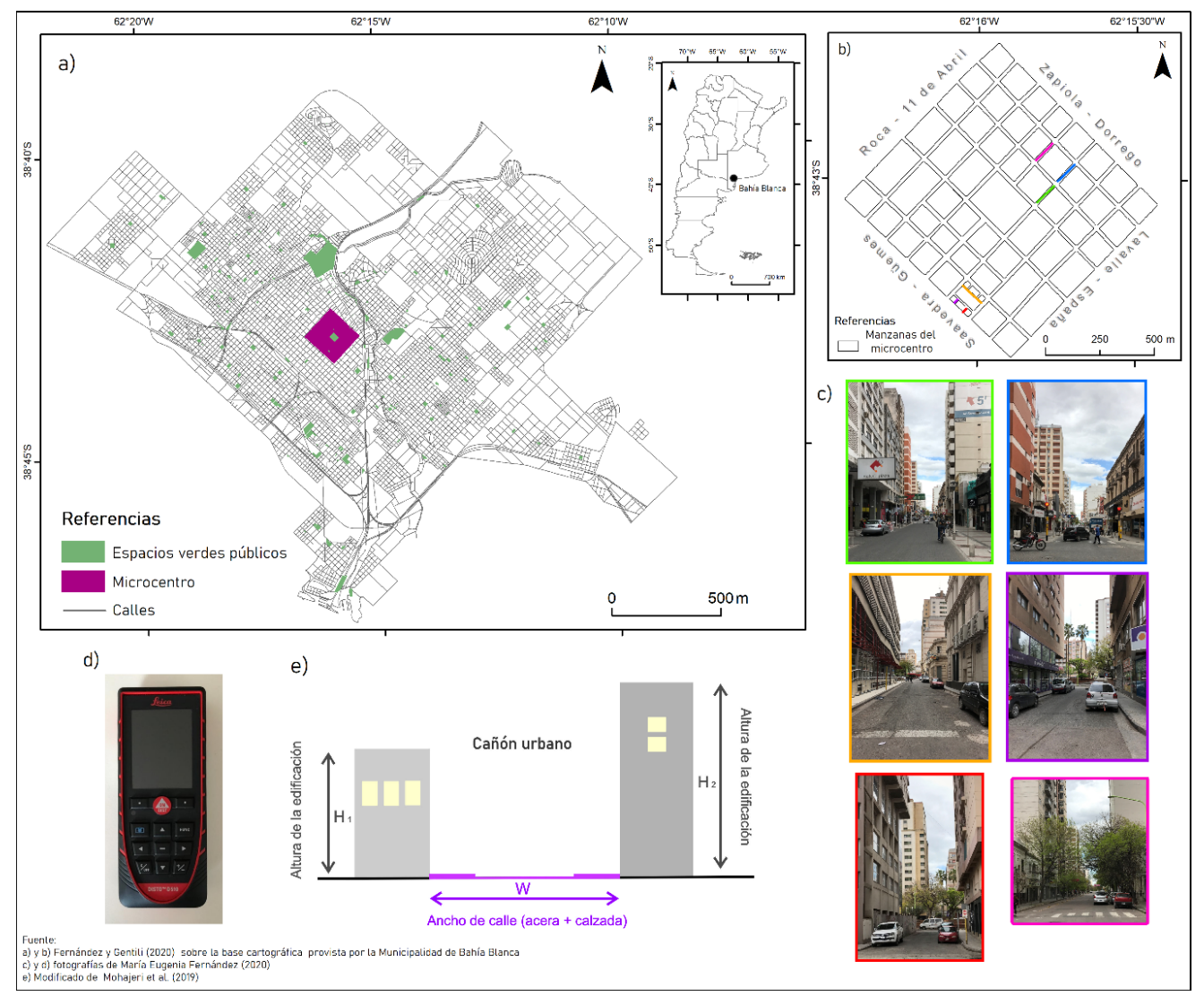

Nota. a) Ciudad de Bahía Blanca; b) Microcentro de Bahía Blanca; c) Cañones urbanos en el microcentro de Bahía Blanca; d) Medidor de distancia láser utilizado en las campañas de medición; e) Características geométricas de los cañones urbanos.

de la energía a escala urbana. En los sectores centrales se manifiesta una marcada preponderancia de flujos de calor sensible y flujos de calor antropogénico (Fernández et al., 2021). Esta distribución calorífica incide en el clima y se relaciona con la ICU y el confort de los habitantes (Capelli de Steffens et al., 2005; Ferrelli, 2016; Oke et al., 2017). En el año 2011, el centro de la ciudad registró menor porcentaje de días confortables (13,6\%) que la zona periurbana (14,8\%) (Fernández et al., 2017; Fernández, Campo et al., 2018).

\section{Técnicas de recolección y análisis de datos}

La presente investigación se llevó a cabo mediante un enfoque cuantitativo. Se planteó el problema y las preguntas de investigación. Se realizó la revisión de la literatura correspondiente, de la cual derivó la confección del marco teórico y la construcción de la hipótesis. La recolección de datos se basó en la medición de características morfológicas del entorno urbano y su posterior análisis estadístico (Hernández Sampieri et al., 2014). Para realizar el análisis a escala local, se recopilaron datos de RSG de Bahía Blanca para el período 2000-2019. Los mismos corresponden a una estación meteorológica marca Davis cuyo sensor de radiación tiene como rango $0-1.800 \mathrm{~W} / \mathrm{m}^{2}$ con una resolución de $1 \mathrm{~W} / \mathrm{m}^{2}$, configurada con un intervalo de registro de 30 minutos. Asimismo, se trabajó con datos de nubosidad provistos por el Servicio Meteorológico Nacional (SMN) y se realizó su análisis estadístico estándar junto con los valores de RSG (W/ $\left./ \mathrm{m}^{2}\right)$. En este sentido, el estudio se circunscribió a los registros de nubosidad diurnos, ya que los registros nocturnos son proclives a poseer subestimaciones (Hahn et al., 1995). Se analizó la frecuencia de ocurrencia de días claros (nubosidad total diaria $\leq 2$ octas) y cubiertos (nubosidad total diaria $\geq 6$ octas) para todo el período. Además, se seleccionaron días $100 \%$ cubiertos (8 octas) de nubes altas, nubes medias y nubes bajas y se calcularon porcentajes de reducción respecto de la RSG recibida en los días claros. Por otro lado, se calcularon porcentajes de ocurrencia de géneros de nubosidad (Cohn, 2017), según estación del año, a fin de vincularlos con las situaciones sinópticas 
más frecuentes de la región. Mediante el software Excel del paquete de Microsoft Office se realizaron los cálculos y los gráficos para contar con información estructural del comportamiento de las variables analizadas.

El cálculo de la insolación directa, difusa y global y la heliofanía efectiva para la ciudad y para el microcentro se realizó con la herramienta Solar Radiation (Spatial Analyst) disponible en el software ArcGIS 10.0, que permite representar cartográficamente la insolación sobre un área geográfica para períodos de tiempo específicos. La herramienta Area Solar Radiation deriva la radiación solar a partir de una superficie ráster. El ráster de superficie de elevación de entrada es un DSM del satélite ALOS PALSAR de 12,5 m de resolución espacial. Mediante la herramienta Extract by mask de Spatial Analyst se recortó el archivo ráster a partir de polígonos correspondientes a Bahía Blanca y al microcentro de la ciudad. Se obtuvieron rásteres referidos a la radiación directa, difusa, solar global y a la heliofanía efectiva. Los valores de radiación solar reflejan la radiación acumulada anual y se expresan en unidades de vatios hora por metro cuadrado $\left(\mathrm{MJ} / \mathrm{m}^{2}\right)$.

Se realizaron campañas urbanas en la que se relevaron elementos referidos a la estructura urbana del microcentro de Bahía Blanca. Se observaron y registraron los anchos de un total de 123 calles y veredas de la ciudad y la altura de la edificación para cada cuadra (Figura 2). Para ello se utilizó un medidor de distancia láser marca LEICA, modelo DISTO D510 (Figura 1d). Su rango de medición de 0,05 hasta $200 \mathrm{~m}$, con una precisión de \pm $1 \mathrm{~mm}$. Paralelamente, se descargaron datos abiertos y colaborativos referidos a la altura de los edificios del microcentro correspondientes a Open Street Maps (OSM) (en formato GeoJSON) desde la web osmbuildings.org y mediante un conversor web fueron transformados a formato shape. Si bien dicha información solo está disponible para una sección del área de interés, fue de utilidad para contrastar y verificar las alturas de los edificios registradas en el campo.

Para calcular la relación de aspecto de cada calle de la ciudad se siguió la metodología empleada por Mohajeri et al. (2019) en su análisis de las características geométricas de cañones urbanos (Figura 1e). En una primera etapa, se calculó la altura de cada edificación de cada cuadra del microcentro, luego el valor medio de la altura de la edificación por cada cuadra y, finalmente, se obtuvo la relación de aspecto (Oke et al., 2017) para cada una de ellas. La relación de aspecto expresa la proporción entre la altura de la edificación (H) y el ancho de las calles (W) (Figura 1e) y se calculó de la siguiente forma:

$$
\text { Relación de aspecto }=\frac{H}{W}
$$

En una segunda etapa se calculó la relación de aspecto media por cada calle. Para ello se promedió la relación de aspecto de ambos lados del cañón urbano (Figura 2). Este análisis permitió cuantificar las características de los cañones urbanos en el microcentro de la ciudad, factor que influye directamente en el comportamiento de las variables climáticas a escala local y microlocal.

\section{Resultados}

\section{Radiación solar global: factores e interacciones} con elementos naturales (nubosidad)

El valor medio de RSG en superficie en la ciudad de Bahía Blanca entre los años 2000 y 2019 fue de 179,09 $\mathrm{W} / \mathrm{m}^{2}$ con una variabilidad interanual de $41,28 \mathrm{~W} / \mathrm{m}^{2}$. El valor anual medio mínimo corresponde al año 2000 con 154,7 W/m² y el máximo al año 2011 con 195,99 W/m². El máximo valor medio (verano) fue de $288,7 \mathrm{~W} / \mathrm{m}^{2}$ y el mínimo valor medio (invierno) fue de $88,0 \mathrm{~W} / \mathrm{m}^{2}$. En primavera y en otoño se hallaron promedios de $213,8 \mathrm{~W} / \mathrm{m}^{2}$ y $141,9 \mathrm{~W} /$ $\mathrm{m}^{2}$, respectivamente. En todas las estaciones térmicas la radiación solar global crece uniformemente desde la salida del sol a lo largo del día, alcanzando su máximo alrededor de 13:00 h (el hecho de que el mediodía solar sea a las 13:00 h obedece a que el huso horario de la Argentina es -3 UTC, aunque el país se halla ubicado en la zona horaria -4 UTC). Durante el verano se alcanzan valores máximos diarios medios de $857,7 \mathrm{~W} / \mathrm{m}^{2}$ y durante el invierno el valor máximo diario medio de RSG es de $372,2 \mathrm{~W} / \mathrm{m}^{2}$. 
Tabla 1

Frecuencia (\%) y valores medio de radiación solar global (W/m2) para días despejados, parcialmente nublados y cubiertos (calculados a partir de observaciones diurnas) según estación del año (período 2000-2019)

\begin{tabular}{|c|c|c|c|c|c|c|}
\hline & \multicolumn{2}{|c|}{ Cielo claro (0-2 octas) } & \multicolumn{2}{|c|}{ Cielo semicubierto (3-5 octas) } & \multicolumn{2}{|c|}{ Cielo cubierto (6-8 octas) } \\
\hline & \multirow{2}{*}{$\begin{array}{l}\text { Número de días } \\
(\%)\end{array}$} & \multirow{2}{*}{$\begin{array}{c}\begin{array}{c}\text { Radiación solar } \\
\text { media }\end{array} \\
(\mathrm{W} / \mathrm{m} 2)\end{array}$} & \multirow{2}{*}{ Número de días (\%) - } & \multirow{2}{*}{$\begin{array}{c}\begin{array}{c}\text { Radiación solar } \\
\text { media }\end{array} \\
(\mathrm{W} / \mathrm{m} 2)\end{array}$} & \multirow{2}{*}{ Número de días (\%) } & \multirow{2}{*}{$\begin{array}{c}\begin{array}{c}\text { Radiación solar } \\
\text { media }\end{array} \\
(\mathrm{W} / \mathrm{m} 2)\end{array}$} \\
\hline & & & & & & \\
\hline Invierno & 29,1 & 182,0 & 34,0 & 148,7 & 37,0 & 93,8 \\
\hline Primavera & 29,2 & 430,6 & 35,1 & 383,4 & 35,7 & 217,6 \\
\hline Verano & 44,6 & 523,0 & 35,2 & 457,8 & 20,2 & 319,4 \\
\hline Otoño & 39,3 & 295,8 & 31,5 & 238,3 & 29,1 & 132,1 \\
\hline Todo el período & 34,9 & 363,5 & 33,9 & 301,4 & 31,1 & 173,3 \\
\hline
\end{tabular}

La Tabla 1 muestra para días despejados, parcialmente nublados y cubiertos su respectiva frecuencia (\%) y valores medios de radiación según estación del año. En Bahía Blanca fueron más frecuentes los días claros, con un porcentaje de ocurrencia del 34,9\%. Mientras que los cielos despejados fueron más frecuentes en otoño y en verano, los cielos cubiertos lo fueron en primavera y en invierno, con porcentajes de 35,7\% y $37 \%$, respectivamente. Los días con cielos claros registraron un valor medio anual de radiación solar de $363,5 \mathrm{~W} / \mathrm{m} 2$, mientras que los días con cielos cubiertos un valor de $173,3 \mathrm{~W} / \mathrm{m}^{2}$, lo que implica reducciones anuales de un $52,3 \%$ entre ambos registros. La tabla muestra que entre días con cielos claros y días con cielos cubiertos se observaron reducciones de entre un $38,9 \%$ en verano y 55,34 \% en otoño. Debido a la probada influencia de las nubes en la RSG, y que es en invierno cuando los días cubiertos son más frecuentes, se destaca la importancia del acceso al sol a escala urbana durante la estación fría. Este análisis pone en evidencia la necesidad de generar políticas públicas y medidas específicas de planeamiento urbano que contemplen el asoleo de espacios públicos, de forma tal que los ciudadanos accedan al sol durante los días despejados de dicha estación. Además, ya que los días despejados son más frecuentes durante la estación cálida, es de especial interés proveer de sombra a los espacios verdes y veredas donde los ciudadanos transitan y/o permanecen durante su tiempo libre.

Se seleccionaron los registros de 8 octas de nubes altas, nubes medias y nubes bajas a los efectos de conocer su capacidad de reducción respecto de la radiación solar global incidente. En el período analizado se registraron nubes altas de los géneros Cirrus (Ci), Cirrocúmulus (Cc) y Cirrostratus (Cs); medias de los géneros Altocúmulus (Ac) y Altostratus (As) y bajas de los géneros Stratocúmulus (Sc), Stratus (St), Cúmulus $(\mathrm{Cu})$ y Cúmulonimbus (Cb) (Cohn, 2017). La Figura 2a muestra los porcentajes de ocurrencia de cada género según la estación del año para Bahía Blanca. Las nubes altas del género Ci son las más frecuentes en todas las estaciones del año, seguido por las Cs y su combinación. En lo que respecta a las nubes medias, se manifestó una preponderancia de As en todas las estaciones. Sc y St son los géneros de nubes bajas más frecuentes en la ciudad. Las $\mathrm{Cb}$ se asocian a procesos convectivos y tormentas eléctricas propias de la estación estival que generan precipitaciones en la región (Zapperi et al., 2007) y presentaron mayor porcentaje de ocurrencia en el verano. La Figura $2 b$ muestra la distribución estacional de la radiación solar en días claros y en días cubiertos. Puede observarse que las nubes bajas y medias son aquellas que mayor capacidad de reducción poseen, con 71,4 \% y 68,4 \% de reducción en todo el período respectivamente. Para ambos tipos de nubes, las reducciones se hacen máximas en las estaciones intermedias. Las nubes altas son las más transparentes respecto de la radiación solar, con reducciones de $35,1 \%$.

Radiación solar: factores e interacciones con elementos antropogénicos

La Figura 3 muestra la distribución espacial de los valores anuales acumulados de la radiación solar (directa, difusa y global) y la heliofanía efectiva (HE) en la ciudad. Se observa la existencia de un gradiente descendente de los valores de radiación (directa, difusa y global) en sentido noreste-suroeste, que se corresponde con el factor altitudinal. Las zonas elevadas ubicadas al noreste de la ciudad presentan valores de radiación (directa, difusa y global) más elevados que la planicie 
Figura 2

Nubosidad y RSG en Bahía Blanca (período 2000-2019)

\begin{tabular}{|c|c|c|c|c|c|}
\hline \multirow{2}{*}{\multicolumn{2}{|c|}{ Tipo/Género }} & Invierno & Primavera & Verano & Otoño \\
\hline & & \multicolumn{4}{|c|}{$\%$} \\
\hline \multirow{4}{*}{ Nubes altas } & $\mathrm{CC}$ & 0,5 & 1,2 & 0,7 & 1,0 \\
\hline & $\mathrm{Cl}$ & 58,5 & 61,9 & 67,8 & 60,7 \\
\hline & $\mathrm{Cl} / \mathrm{CS}$ & 16,6 & 18,8 & 15,8 & 20,4 \\
\hline & cs & 24,4 & 18,0 & 15,8 & 17,9 \\
\hline \multirow{2}{*}{ Nubes medias } & $A C$ & 92,2 & 91,1 & 95,9 & 89,0 \\
\hline & AS & 7,8 & 8,9 & 4,1 & 11,0 \\
\hline \multirow{6}{*}{ Nubes bajas } & $\mathrm{CB}$ & 1.7 & 8,0 & 18,7 & 4,9 \\
\hline & $\mathrm{CU}$ & 2,8 & 15,3 & 23,7 & 11,6 \\
\hline & $\mathrm{CU} / \mathrm{sc}$ & 2,0 & 3,6 & 4,6 & 1,8 \\
\hline & $\mathrm{CU} / \mathrm{ST}$ & 0,9 & 1,0 & 0,5 & 1,0 \\
\hline & SC & 54,0 & 45,9 & 41,2 & 55,4 \\
\hline & ST & 38,6 & 26,2 & 11,4 & 25,4 \\
\hline
\end{tabular}

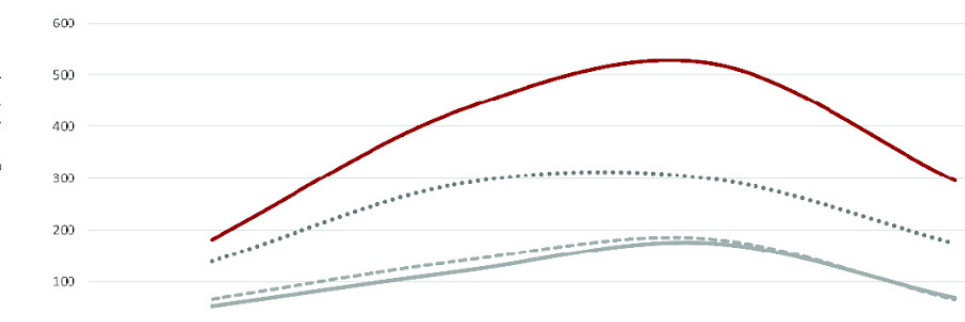

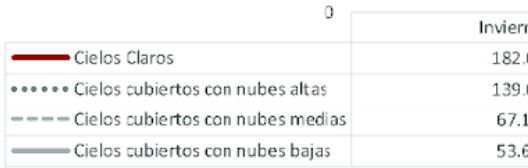

Nota. a) Ocurrencia (\%) de géneros nubosos según estación del año; b) Distribución estacional de RSG (W/m2) en cielos claros, con nubes altas, medias y bajas en Bahía Blanca.

Figura 3

Distribución espacial de la radiación solar (directa, difusa y global) y la HE en Bahía Blanca
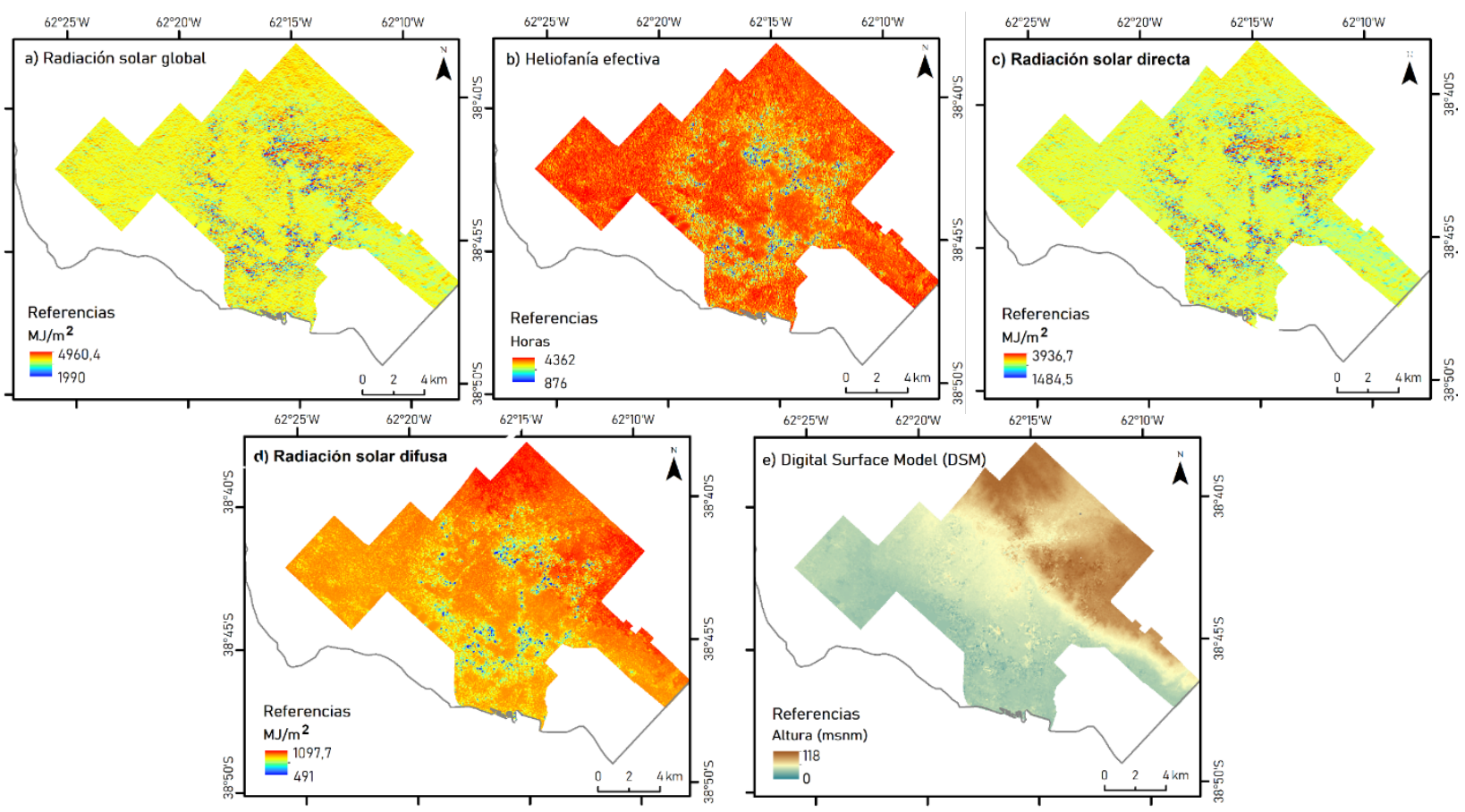

Nota. a-d) Distribución espacial de la radiación solar directa, difusa y global y la HE en Bahía Blanca; e) DSM de la ciudad de Bahía Blanca. 
Figura 4

Espacios verdes públicos, vegetación y descriptores morfológicos urbanos en el microcentro de Bahía Blanca
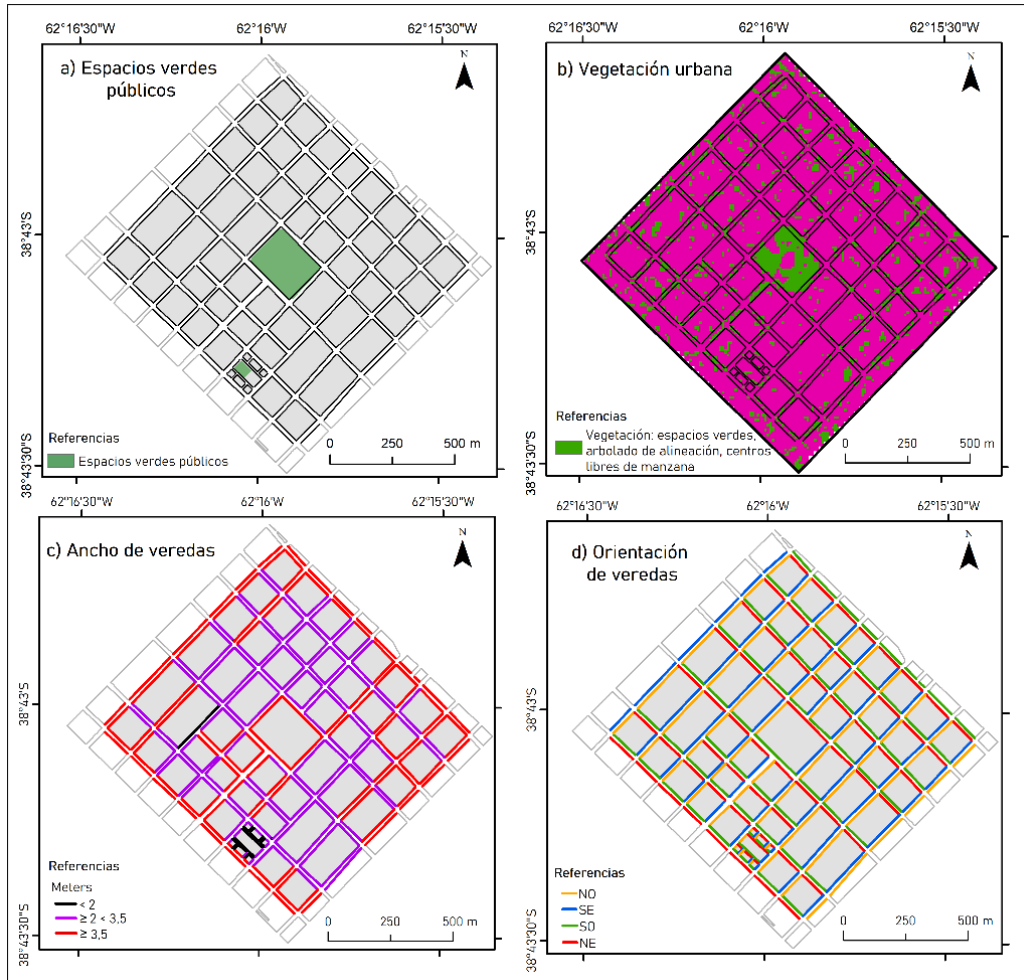

Nota. a) Espacios verdes públicos; b) vegetación urbana; c) ancho; d) orientación de veredas en el microcentro de Bahía Blanca.

baja cercanas a la costa. Por otra parte, se manifiesta la influencia de la estructura urbana en el patrón radiativo. Los valores mínimos de radiación solar (Figuras 3a, 3c, 3d) y heliofanía efectiva (Figura 3b) en Bahía Blanca se localizan en el microcentro de la ciudad y en los grandes espacios verdes, donde se ubica el arbolado urbano de mayor magnitud, por lo que puede deducirse que las sombras proyectadas por los edificios y árboles definen el acceso al sol.

Debido a que la estructura urbana posee directa incidencia en la radiación solar al interior de la ciudad y es la zona central una de las que presenta la mayor variabilidad en la distribución de la variable, se analizaron diversas características de la estructura del microcentro (Figura 1b). La Figura 4 muestra la distribución espacial de algunos de los principales descriptores morfológicos urbanos que poseen influencia en la radiación. La vegetación urbana se concentra fundamentalmente en los espacios verdes del área, así como también en las veredas (arbolado de alineación) y en los centros de manzana. En la ciudad, los ejemplares arbóreos permitidos en veredas dependen fundamentalmente del ancho de esta (Figura 4c). Al respecto, la Ordenanza Municipal N 16.859 (Modificación Ordenanza 15.523: Arbolado Urbano y Espacios Verdes, 2012) define que en las veredas de anchos mayores o iguales a $2 \mathrm{~m}$ y menores a 3,5 $\mathrm{m}$ las especies a implantar serán de tercera magnitud (hasta $10 \mathrm{~m}$ ), mientras que en aquellas de más de 3,5 $\mathrm{m}$ de ancho las especies a implantar serán de primera magnitud (más de $20 \mathrm{~m}$ ) y segunda magnitud (10 a $20 \mathrm{~m}$ de altura) (Duval y Benedetti, 2017). Debido a que la magnitud de la especie define la altura que alcanzará el ejemplar arbóreo en la edad adulta (y, por lo tanto, la sombra que proyectará), los anchos de veredas son un factor de interés en el análisis de la radiación a escala microlocal. La Figura 4c muestra que gran parte de las veredas del microcentro poseen un ancho inferior a 3,5 m. Asimismo, al comparar los mapas de las Figuras $4 \mathrm{~b}$ y $4 \mathrm{c}$ se observa que el arbolado de alineación se desarrolla fundamentalmente en las veredas más anchas y alrededor de la plaza central, donde la variable solar se verá directamente afectada por su sombra. Además, se analizó la orientación de la trama urbana (Figura 4d), es decir, la consiguiente 
Figura 5

Descriptores morfológicos urbanos del microcentro de Bahía Blanca

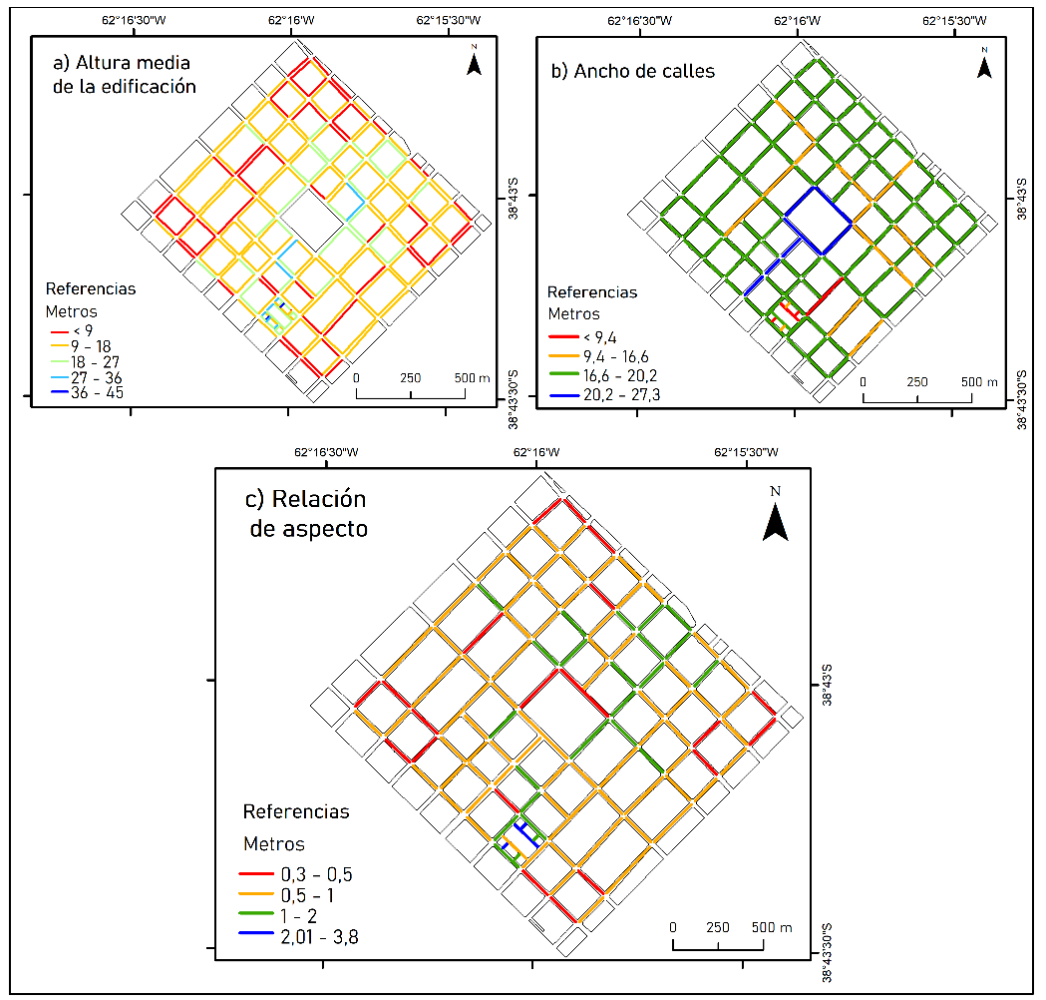

Nota. a) Altura media de la edificación; b) Ancho de calles; c) Relación de aspecto de los cañones urbanos en el microcentro.

disposición del parque edificado respecto de los puntos cardinales. Por su forma de damero, la trama urbana del microcentro de Bahía Blanca posee fachadas edilicias con orientaciones noreste, noroeste, suroeste y sureste. Las fachadas orientadas al noroeste y noreste poseen mayor posibilidad de exposición a los rayos directos del sol y, por lo tanto, mayor grado de influencia en el balance térmico de los edificios y en el confort de los transeúntes de acuerdo con la estación del año.

Por otro lado, se analizó la altura de los edificios, el ancho de las calles (extensión que contempla a ambas aceras y la calzada) y la relación existente entre ambos parámetros (relación de aspecto). La Figura 5 muestra que la altura media de la edificación en el microcentro oscila alrededor de los 5,33 $\mathrm{m}$ y la altura máxima alcanza los $45 \mathrm{~m}$. En la mayor parte de las cuadras del microcentro la altura media de la edificación es entre 9 y $18 \mathrm{~m}$. Las mayores alturas se registran en las cercanías de la plaza central, alcanzando hasta $36 \mathrm{~m}$. El ancho de las calles define la proximidad física entre dos edificaciones enfrentadas. Las calles del microcentro presentan un ancho variable: menos de $10 \mathrm{~m}$ en los sectores más angostos y más de $27 \mathrm{~m}$ en los más anchos. Las calles más anchas se observan alrededor de la plaza central, donde se midieron anchos de entre $9 \mathrm{~m}$ y 20,2 m. Los dos parámetros anteriores también fueron considerados juntos, sintetizados en la relación de aspecto (aspect ratio) de los cañones urbanos. Se calculó la relación de aspecto media para cada una de las cuadras (Figura 5), ya que dicho parámetro define en gran parte la cantidad de energía recibida por los edificios y al nivel del suelo. En la medida en que la relación de aspecto de los cañones urbanos disminuye, la cantidad de energía recibida a nivel de suelo aumenta. Se hallaron cañones urbanos poco profundos (relación de aspecto $<1$ ), uniformes (relación de aspecto cercano a 1) y profundos (relación de aspecto > 2) (Shishegar, 2013).

La Figura 6 muestra la distribución de la radiación solar en el microcentro, que se ve fuertemente determinada por los descriptores morfológicos urbanos antes mencionados. En todos los casos los valores se refieren a un valor acumulado anual (año 2018). La RSG (Figura 6a) es menor 
Figura 6

Distribución espacial de la radiación solar (directa, difusa y global) y la HE en el microcentro de Bahía Blanca

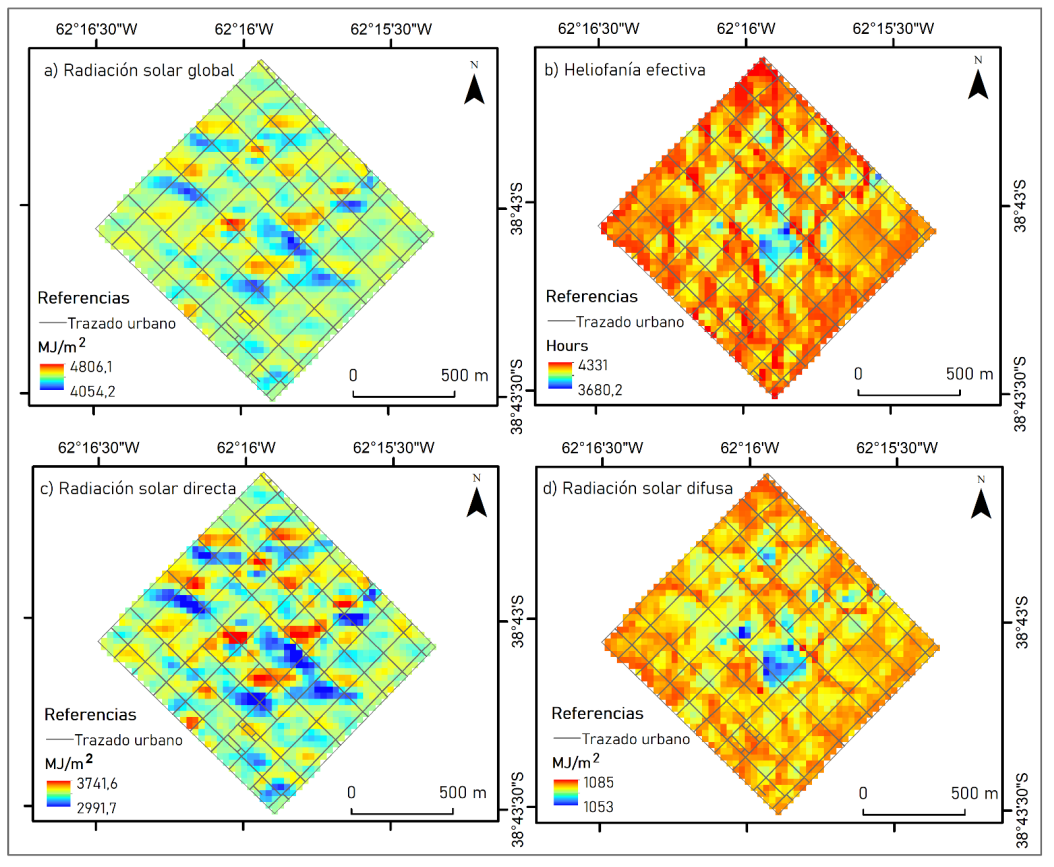

Nota. a) Radiación solar global; b) Heliofanía efectiva; c) Radiación solar directa; d) Radiación solar difusa.

en las zonas donde la edificación es más alta y compacta, que coinciden con la localización de los cañones urbanos más profundos (Figuras 5 y 6). Asimismo, la presencia de vegetación arbórea en el sector central, que coincide con la plaza Rivadavia, influye en los valores de RSG modelados y se evidencia una diferenciación en la $\mathrm{RSG}$ recibida en los centros de manzana respecto de los bordes de estas (Figuras 4 y 5). En las veredas con orientación noroeste o noreste (Figura 4d), los valores de radiación son mayores que en los centros de manzana. En lo que respecta a la radiación solar directa (Figura 6c), se observa una máxima variabilidad espacial en su distribución, con un rango máximo de $750 \mathrm{MJ} / \mathrm{m} 2$. Valores máximos y mínimos varían sustancialmente en distancias pequeñas. Puede inferirse que las sombras de las edificaciones afectan directamente a esta variable. Los menores valores se localizan en los centros de manzana y en los sectores ubicados en las cercanías de la plaza central, y los máximos valores en áreas puntuales del microcentro y, principalmente, en las calles y veredas. La radiación solar difusa (Figura $6 \mathrm{~d}$ ) posee menor variabilidad que la directa en esta escala, con valores mínimos en las cercanías de la plaza central. En lo que respecta a la HE (Figura 6b), esta posee una marcada variabilidad en los sectores centrales del microcentro. Las zonas centrales presentan valores mínimos, que coinciden con aquellas donde la radiación solar global es menor. Las áreas cercanas a las calles y ciertos sectores de los centros de manzana presentan los mayores valores de HE del área analizada.

El análisis de la estructura urbana y la dinámica de la radiación solar en el microcentro es un insumo fundamental para tener en cuenta a la hora de fortalecer la infraestructura verde en el área. La Figura 7 muestra las zonas aptas para la plantación de especies arbóreas en el microcentro de la ciudad según el ancho de las veredas, su orientación y la relación de aspecto de los cañones urbanos. En primer término, se identificaron aquellas veredas con más de $2 \mathrm{~m}$ de ancho (Modificación Ordenanza 15.523: Arbolado Urbano y Espacios Verdes, 2012), con orientación noroeste o noreste y con una relación de aspecto de los cañones urbanos menor a $1 \mathrm{~m}$ (cañones bajos). Estas condiciones favorecen el alcance de radiación solar directa, factor fundamental para el desarrollo de la vegetación. En la figura es posible identificar dos categorías de veredas aptas para la plantación de árboles. En aquellas de ancho mayor o igual a 2 m y menor a 3,5 m, la Ordenanza Municipal No 16.859 (Modificación Ordenanza 15523: Arbolado Urbano y Espacios Verdes, 2012) estipula la plantación de especies de tercera dimensión, con una distancia 
Figura 7

Propuestas de reforestación en el microcentro según ancho de veredas, orientación y relación de aspecto



mínima de $4 \mathrm{~m}$ y máxima de $5 \mathrm{~m}$ entre árboles. Por otro lado, en aquellas veredas con un ancho de 3,5 $\mathrm{mo}$ más, se estipula la plantación de especies de primera y segunda dimensión, con una distancia mínima de $6 \mathrm{~m}$ y máxima de $8 \mathrm{~m}$ entre árboles.

\section{Discusión}

Se analizó la distribución espaciotemporal de la radiación solar en Bahía Blanca y su vinculación con elementos naturales y antropogénicos en la ciudad. Los hallazgos relacionados con la radiación solar global y las coberturas nubosas coinciden con lo expuesto por investigaciones previas referidas a las coberturas nubosas en Bahía Blanca y en el sur de la provincia de Buenos Aires y a sus características de clima continental (Fernández et al., 2015; Fernández, Gentili et al., 2018). Los cielos despejados fueron más frecuentes en otoño y en verano y los cielos cubiertos lo fueron en primavera y en invierno. Entre días con cielos claros y días con cielos cubiertos se observaron reducciones de entre un 38,9\% en verano y $55,34 \%$ en otoño. Estos valores coinciden con los expuestos por otros investigadores en el análisis de la influencia de las coberturas nubosas en la radiación solar en Bahía Blanca (para otros períodos) (Fernández et al., 2015) y en otros lugares del mundo (El-Nouby Adam, 2011; Sánchez et al., 2012). Las nubes bajas y medias son aquellas que mayor capacidad de reducción de la RSG poseen, con porcentajes de 71,4 \% y 68,4 \% respectivamente. Las nubes altas son las más transparentes respecto de la RSG, con reducciones menores al $40 \%$.

El estudio permitió verificar que el acceso al sol como recurso (para su aprovechamiento energético, disfrute y elemento fundamental para la salud y el confort) se ve afectado en la ciudad y que la estructura urbana es uno de los principales elementos que influyen en su variabilidad espacial a escala local. Se verificó que la geometría de los cañones urbanos y su orientación definen en el microcentro la disponibilidad del recurso solar en aceras y calzadas. Estos hallazgos son consistentes con lo expuesto por otros investigadores en el análisis de los cañones urbanos y la dinámica radiactiva a escala local. Entre ellos, Li y Ratti (2018) afirmaron que los cañones urbanos del centro de Boston tienen menores valores de heliofanía efectiva y radiación solar en comparación con otras regiones del área de estudio. Respecto de la relevancia de la orientación de la trama urbana, Mohajeri et al. (2019) afirmaron que la orientación calle-cañón es el descriptor morfológico urbano con mayor efecto sobre la radiación solar recibida, tanto para las superficies de las calles como para las fachadas de los edificios. En relación con ello, Garcia-Nevado et al. (2016) documentaron que en Barcelona (España) las intersecciones de las calles conducen a un aumento del potencial solar vertical, que es más significativo en aquellas superficies que miran al SE o SO para el solsticio de invierno $\left(+1,3 \mathrm{kWh} / \mathrm{m}^{2}\right.$ por día) y aquellas que miran al $\mathrm{E} \mathrm{u} \mathrm{O}$ para el solsticio de verano. 
Asimismo, la presencia de vegetación arbórea en el sector central, que coincide con la plaza Rivadavia, influye en los valores de RSG modelados. La influencia del arbolado urbano sobre la insolación recibida ha sido también documentada por otros autores. Por ejemplo, Arboit y Betman (2014) registraron una disponibilidad del recurso solar de solo un $15 \%$ en ciertos sectores del Área Metropolitana de Mendoza, Argentina. Por su parte, Lotufo Bueno-Bartholomei y Labaki (2003) estudiaron la influencia de las especies arbóreas más frecuentes en Campinas (Brasil) en la radiación solar recibida y hallaron reducciones de ente 29,9\% y 92,8 \%. Se identificaron veredas aptas para plantación de ejemplares arbóreos en el microcentro, de acuerdo con el ancho de la acera, la orientación y relación de aspecto. En lo que respecta al fortalecimiento de la infraestructura verde, Gentili et al. (2020) propusieron medidas a ser implementadas en las playas de estacionamiento del microcentro.

\section{Conclusiones}

Se analizó la distribución espaciotemporal de la radiación solar en Bahía Blanca y su vinculación con elementos naturales y antropogénicos en la ciudad. Este trabajo permitió verificar que la nubosidad, la morfología urbana y la vegetación arbórea son los principales factores que determinan la distribución de la RSG a escala urbana. Se generaron conocimientos relativos al clima solar en la ciudad que serán un insumo de interés al proponer medidas locales de desarrollo sostenible, lo que incluye el fortalecimiento de la infraestructura verde urbana.

En lo que respecta a la vinculación de la radiación solar con elementos naturales, se verificó que la nubosidad define la distribución de la RSG en la ciudad. El estudio demostró que los días con cielos claros registraron un valor anual de radiación solar de $363,5 \mathrm{~W} / \mathrm{m}^{2}$, mientras que los cielos cubiertos un valor de $173,3 \mathrm{~W} / \mathrm{m}^{2}$, lo que implica reducciones anuales de un 52,3\% entre ambos registros. Las nubes bajas y medias son aquellas que mayor capacidad de reducción de la RSG poseen, con porcentajes de 71,4 \% y 68,4 \%, respectivamente. Las nubes altas son las más transparentes respecto de la RSG, con reducciones menores al $40 \%$.
En lo referido a la radiación solar y los elementos antropogénicos, se analizó la distribución espacial de algunos de los principales descriptores morfológicos urbanos del microcentro que poseen influencia en la radiación: ancho y orientación de veredas, ancho de calles y altura de la edificación. Se hallaron cañones urbanos poco profundos, uniformes y profundos. La distribución de la RSG en el microcentro muestra valores más bajos en las zonas donde la edificación es más alta y compacta, donde los cañones urbanos son más profundos. Asimismo, en las veredas con orientación noroeste o noreste los valores de radiación son mayores que en los centros de manzana. El estudio permitió verificar que el acceso al sol como recurso (para su aprovechamiento energético, disfrute y elemento fundamental para la salud y el confort) se ve afectado principalmente por la estructura urbana de Bahía Blanca.

La compacidad edilicia es uno de los principales elementos que influyen en la variabilidad espacial del recurso a escala local. Los valores mínimos de radiación solar y heliofanía efectiva en Bahía Blanca se localizan en el microcentro y en los grandes espacios verdes, donde se encuentra el arbolado urbano de mayor magnitud. Por lo tanto, puede deducirse que las sombras proyectadas por los edificios y árboles definen el acceso al sol.

Este análisis permitió identificar posibles medidas urbanísticas concretas para consolidar ciudades sostenibles y resilientes y es un paso importante para contribuir a la mitigación de las problemáticas urbanas más comunes y relevantes. Conocer la disponibilidad y distribución temporal de la radiación solar en la cuidad es un avance importante en materia de estimación del potencial energético renovable urbano. Asimismo, debido a que las nubes tienen gran capacidad de absorción y reflexión de energía, la nubosidad es un elemento del clima de interés para ser considerado en el planeamiento urbano. En este sentido, se destaca la importancia del acceso al sol a escala urbana durante la estación fría.

El estudio evidenció la necesidad de generar políticas públicas y medidas específicas de planeamiento que 
contemplen el asoleo de espacios públicos (como, por ejemplo, regulación de la morfología urbana o plantación de especies arbóreas caducas, entre otras), de forma tal que los ciudadanos accedan al sol durante los días despejados invernales. Además, ya que los días despejados son más frecuentes durante la estación cálida, es de interés proveer de sombra a los espacios verdes y veredas donde los ciudadanos transitan y/o permanecen durante su tiempo libre. Al respecto, se identificaron veredas aptas para plantación de ejemplares arbóreos en el microcentro, de acuerdo con el ancho de la acera, la orientación y relación de aspecto.

De los tres factores analizados con influencia en la radiación solar (la nubosidad, la morfología urbana y la vegetación arbórea) son los dos últimos aquellos susceptibles a ser intervenidos desde el planeamiento urbano. $\mathrm{Al}$ respecto, dichos factores poseen temporalidades diferenciales tanto en su aplicación como en los efectos deseados, variables que deben ser consideradas al momento de plantear medidas de planeamiento urbano. El presente trabajo constituye un avance de interés en la temática y un estudio inédito en Bahía Blanca, cuyos métodos pueden ser utilizados por urbanistas a la hora de diseñar e implementar estrategias urbanas sostenibles en otras ciudades.

\section{Referencias bibliográficas}

Abal, G. (2012). Fundamentos de energía solar. Universidad de la República.

Acero Alejandro, J. A. (2010). Influencia de la vegetación en la calidad del aire y el clima urbano. Congreso Nacional de Medio Ambiente CONAMA, 1-13. http://www.upv.es/ contenidos/CAMUNISO/info/U0644000.pdf

Arabi, R., Shahidan, M. F., Kamal, M. S. M., Fakri Zaky Bin Ja'afar, M., \& Rakhshandehroo, M. (2015). Mitigating Urban Heat Island Through Green Roofs. Current World Environment, 10(1), 918-927.https://doi.org/10.12944/CWE.10.Special-Issue1.111
Arboit, M. E. (2013). Permeabilidad del arbolado urbano a la radiación solar: estudio de dos especies representativas en entornos urbanos de baja densidad del Área Metropolitana de Mendoza, Argentina. Hábitat Sustentable, 3(2), 3-18. http://revistas.ubiobio.cl/index.php/RHS/article/view/431

Arboit, M. E. y Betman, E. (2014). Radiación solar en entornos urbanos forestados con clima seco. Revista de Urbanismo, (31), 76-94. https://doi.org/10.5354/0717-5051.2014.29425

Arboit, M. E., \& Betman, E. (2017). Comparative Study of Solar Radiation Availability in Dry Climate Urban Environment Forested Areas, in Mendoza, Argentina. International Journal Environmental Science and Sustainable Development, 1(1), 1-13. https://doi.org/10.21625/essd.vli1.16

Bajsanski, I., Stojakovic, V., \& Jovanovic, M. (2016). Effect of tree location on mitigating parking lot insolation. Computers, Environment and Urban Systems, 56, 59-67. https://doi.org/10.1016/J.COMPENVURBSYS.2015.11.006

Basso, M., Arboit, M. E., Mesa, A. y De Rosa, C. (2013). Desarrollo e implementación de indicadores morfológicos para ser incorporadosal Código Urbanoy de Edificación del municipio de Godoy Cruz, en el Área Metropolitana de Mendoza. Energías Renovables y Medio Ambiente, (31), 59-66. http:// repositorioslatinoamericanos.uchile.cl/handle/2250/1903309

Benedetti, G. M. y Campo de Ferreras, A. M. (2007). Arbolado de alineación: el mapa verde un barrio en la ciudad de Bahía Blanca, Argentina. Papeles de Geografía, (45-46), 27-38. https://revistas.um.es/geografia/article/view/43081

Benedetti, G. M., Duval, V. S., Campo, A. M. y Barrionuevo, L. (2013). El aeropolen en la ciudad de Bahía Blanca (Argentina): aportes para la gestión del arbolado público desde la selección de especies. Revista de la Asociación Argentina de Ecología de Paisajes, 4(2), 199-210. http:// ri.conicet.gov.ar/handle/11336/12452 
Blanchard, C. L., \& Tanenbaum, S. J. (2003). Differences between Weekday and Weekend Air Pollutant Levels in Southern California. Journal of the Air and Waste Management Association, 53(7), 816-828. https://doi.or $\mathrm{g} / 10.1080 / 10473289.2003 .10466222$

Bróndolo, M., Campos, M., Zinger, S., Del Pozo, O. y Lorda, M. A. (1994). Geografía de Bahía Blanca. Ediciones Encestando.

Cabrera, A. (1976). Enciclopedia argentina de agricultura y jardinería: regiones fitogeográficas argentinas. Acme.

Campo de Ferreras, A. M., Capelli de Steffens, A. M. y Diez, P. (2004). Clima del Suroeste bonaerense. Departamento de Geografía y Turismo, Universidad Nacional del Sur.

Capelli de Steffens, A. M., Campo de Ferreras, A. M. y Gil, V. (2003). Condiciones climáticas generadas en el área céntrica de la ciudad de Bahía Blanca. Contribuciones Científicas GAEA, 15, 125-130.

Capelli de Steffens, A. M., Piccolo, M. C. yCampo de Ferreras, A. M. (1989). La circulación del aire en torno a complejos edilicios bahienses. Revista Universitaria de Geografía, 4, 39-53.

Capelli de Steffens, A. M., Piccolo, M. C. y Campo de Ferreras, A. M. (2005). Clima urbano de Bahía Blanca. Dunken.

Carrasco Aldunate, C. (2010). Morfología y microclima urbano análisis de la forma espacialy materiales como modeladores del microclima de tejidos urbanos mediterráneos costeros: el caso de la ciudad de Valparaíso [Tesis de doctorado, Universitat Politécnica de Catalunya]. En TDX (Tesis Doctorals en Xarxa). http://www.tdx.cat/handle/10803/51572

Cohn, S. A. (2017). International Cloud Atlas. Manual on the Observation of Clouds and Other Meteors. WMO.

Coutts, A. M., \& Harris, R. (2013).A multi-scale assessment of urban heating in Melbourne during an extreme heat event and policy approaches for adaptation. Victorian Centre for Climate Change Adaption Research. https://watersensitivecities.org.au/ content/vcccar-report-multi-scale-assessment-urban-heatingmelbourne-extreme-heat-event-policy-approaches-adaptation/
Curreli, A. (2017). La integración de la radiación solar en la ciudad compacta [Tesis de Maestría, Universitat Politècnica de Catalunya]. Repositorio institucional. https://wwwaie. webs.upc.edu/maema/wp-content/uploads/2016/06/08_ Alessandra-Curreli_Integracion-de-la-radiacion-solar-enun-tejido-urbano-compacto_COMPLETO2.pdf

Curreli, A., Serra-Coch, G., Isalgue, A., Crespo, I., \& Coch, H. (2016). Solar Energy as a Form Giver for Future Cities. Energies, 9(7), 544. https://doi.org/10.3390/en9070544

Declet-Barreto, J., Knowlton, K., Jenerette, G. D., Buyantuev, A., Declet-Barreto, J., Knowlton, K., Jenerette, G. D., \& Buyantuev, A. (2016). Effects of Urban Vegetation on Mitigating Exposure of Vulnerable Populations to Excessive Heat in Cleveland, Ohio. Weather, Climate, and Society, 8(4), 507-524. https://doi.org/10.1175/WCAS-D-15-0026.1

Dugarova, E., \& Gülasan, N. (2017). Global Trends. Challenges and opportunities in the implementation of the Sustainable Development Goals. United Nations Research Institute for Social Development, UNRISD. https://www.unrisd. org/unrisd/website/document.nsf/.../

Duval, V. S.yBenedetti, G. (2017). Diagnóstico del arbolado público lineal del entorno de la Universidad Nacional del Sur. En F. I. Contreras(Ed.), Junta de Geografía de la Provincia de Corrientes (pp. 5-17). Junta de Geografía de la Provincia de Corrientes. https://www.researchgate.net/publication/324223505

El-Nouby Adam, M. (2011). Effect of Macrophysical Parameters of Clouds on Broadband Solar Radiation (295-2800 nm) at a Subtropical Location. Atmospheric and Oceanic Science Letters, 4(3), 181-185. https://doi.org/10.1080/16 742834.2011.11446926

Ercolani, P. (2005). Configuración socio-espacial urbana: el espacio del ocio en Bahía Blanca. Estado actual y propuesta de futuro. Universitat de les Illes Balears.

European Environment Agency. (2015). Urban sustainability issues - What is a resource-efficient city? (Número EEA Technical Report No 23/2015 Urban). Edición del autor. https://doi.org/10.2800/389017 
Fernández, M. E. (2020). La radiación solar en Bahía Blanca. Universidad Nacional del Sur.

Fernández, M. E., Campo, A. M. y Gentili, J. O. (2015). Comportamiento temporal de la radiación solar global en la ciudad de Bahía Blanca, Argentina. Revista de Climatología, (15), 51-64. https://dialnet.unirioja.es/servlet/ articulo?codigo $=7416385$

Fernández, M. E., Campo, A. M. y Gentili, J. O. (11-13 de abril de 2018). Análisis bioclimatológico en la ciudad de Bahía Blanca, Argentina [Sesión de conferencia]. XII Jornadas Nacionales de Geografía Física "Contribuciones de la Geografía Física a la gestión de los territorios”. Universidad Nacional de la Patagonia San Juan Bosco - Red Argentina de Geografía Física Trelew, Chubut, Argentina. https:// www.researchgate.net/publication/330967411_ Analisis_bioclimatologico_en_la_ciudad_de_Bahia_ Blanca_Argentina

Fernández, M. E., \& Gentili, J. O. (2021). Radiación solar en entornos urbanos: un recurso, un peligro y un derecho. Análisis desde la percepción en Bahía Blanca, Argentina. Estudios Geográficos, 82(291), e076. https://doi.org/https:// doi.org/10.3989/estgeogr.202187.087

Fernández, M.E., Gentili, J. O.yCampo,A. M. (2017). Caracterización bioclimática media de la ciudad de Bahía Blanca, Arg. En Asociación de Universidades Grupo Montevideo (AUGM) \& Universidad Nacional de Itapúa (UNI) (Eds.), XXV Jornadas de Jóvenes Investigadores. Edición del autor.

Fernández, M. E., Gentili, J. O., \& Campo, A. M. (2018). Sunshine Duration Analysis as a First Step to Estimate Solar Resource for Photovoltaic Electricity Production in Middle Latitudes. Environmental Processes, 5(2), 313-328. https://doi.org/10.1007/s40710-018-0298-3

Fernández, M. E., Picone, N., Gentili, J. O., \& Campo, A. M. (2021). Analysis of the Urban Energy Balance in Bahía Blanca (Argentina). Urban Climate, 37, 100856. https:// doi.org/https://doi.org/10.1016/j.uclim.2021.100856
Ferrelli, F. (2016). Análisis del clima local y micro-local de la ciudad deBahía Blanca [Tesis dedoctorado, Universidad Nacional del Sur]. Repositorio institucional. http://repositoriodigital.uns. edu.ar/bitstream/123456789/2698/1/Tesis_Ferrelli_2016.pdf

Fittipaldi, R. Á., Espasa, L., Masrandrea, A. y Michalijos, M. P. (2018). Geografía de Bahía Blanca. La conformación del espacio urbano en el siglo XX. En M. N. Cernadas y J. B. Marcilese (Eds.), Bahía Blanca siglo XX: historia política, económica y sociocultural (pp. 15-36). EdiUNS.

Formiga, N. y Marenco, S. (2000). La dinámica urbana. El proceso de desarrollo vertical y la problemática de la marginalidad urbana en Bahía Blanca. EdiUNS.

Garcia-Nevado, E., Pages-Ramon, A., \& Coch, H. (2016). Solar Access Assessment in Dense Urban Environments: The Effect of Intersections in an Urban Canyon. Energies, 9(10), 756. https://doi.org/10.3390/en9100796

Gasparrini, A., Guo, Y., Hashizume, M., Lavigne, E., Zanobetti, A., Schwartz, J., Tobias, A., Tong, S., Rocklöv, J., Forsberg, B., Leone, M., De Sario, M., Bell, M. L., Guo, Y.-L. L., Wu, C., Kan, H., Yi, S.-M., de Sousa Zanotti Stagliorio Coelho, M., Saldiva, P. H. N., ... Armstrong, B. (2015). Mortality risk attributable to high and low ambient temperature: A multicountry observational study. The Lancet, 386(9991), 369-375. https://doi. org/10.1016/S0140-6736(14)62114-0

Gentili, J. O. (2005). Problemáticas asociadas a las playas de estacionamiento en el microcentro de Bahía Blanca (Documento inédito).

Gentili, J. O., Fernández, M. E., \& Gil, V. (2020). Influence of Topography on Local Atmospheric Features in a Peri-urban Area of Bahía Blanca (Argentina). Environmental Processes, 7(1), 23-40. https://doi.org/10.1023/A:1020824109575

Gentili, J. O., Fernández, M. E., Ortuño Cano, M. de los Á., \& Campo, A. M. (2020). Assessment of the sustainable potential of parking lots in Bahía Blanca City, Argentina. GeoJournal, (85), 1257-1275. https://doi.org/https://doi. org/10.1007/s10708-019-10021-5 
Gill, S. E., Handley, J. F., Ennos, A. R., \& Pauleit, S. (2007). Adapting cities for climate change: the role of the green infrastructure. Built Environment, 33(1), 155-133. http:// urbanspace.rec.org/files/Article_Gill_Adapting_Cities_for_ CC.pdf

Goodrich, E. (2016). Measuring the effects of parking lots and tree shading on microscale urban heat islands [Tesis de Licenciatura, University of Oregon]. Repositorio institucional. https://scholarsbank.uoregon.edu/xmlui/ bitstream/handle/1794/20288/FINAL Thesis-Goodrich. pdf?sequence $=1$

Gray Lovio, O. R., Abreu Daniel, A., Bonito Lovio, D., Díaz González, O.y Martínez Chapman, E. (2014). Fotoeducación: información básica. Revista Cubana de Medicina General Integral, 30(4), 481-486. http://scielo.sld.cu/ scielo.php?script=sci_arttext\&pid=S086421252014000400009

Gronlund, C. J., Zanobetti, A., Wellenius, G. A., Schwartz, J. D., \& O’Neill, M. S. (2016). Vulnerability to renal, heat and respiratory hospitalizations during extreme heat among U.S. elderly. Climatic Change, 136(3-4), 631-645. https:// doi.org/10.1007/s10584-016-1638-9

Habte, A., Stoffel, T., Perez, R., Myers, D., Gueymard, C., Blanc, P., \&Wilbert, S. (2017). Overview of Solar Radiation Resource Concepts. En M. Sengupta, A. Habte, C. Gueymard, S. Wilbert, D. Renné, \& T. Stoffel (Eds.), Best Practices Handbook for the Collection and Use of Solar Resource Data for Solar Energy Applications (Second Edition) (pp. 2.1-2.22). NREL. https://www.nrel.gov/docs/fy18osti/68886.pdf

Hahn, C. J., Warren, S. G., \& London, J. (1995). The Effect of Moonlight on Observation of Cloud Cover at Night, and Application to Cloud Climatology. Journal of Climate, 8(5), 1429-1446. https://doi.org/10.1175/15200442(1995)008<1429:TEOMOO>2.0.CO;2

Hermosillo Villalobos, J. J., Gudiño Ayala, D. y Mendoza Ramírez, M. (1995). Notas sobre el curso de Energía Solar. Instituto Tecnológico y de Estudios Superiores de Occidente. http://www.solartronic.com/download/ curso_iteso.pdf
Hernández Sampieri, R., Fernández Collado, C. y Baptista Lucio, P. (2014). Metodología de la investigación. McGraw-Hill.

Hwang, R.-L., Lin, T.-P., \& Matzarakis, A. (2011). Seasonal effects of urban street shading on long-term outdoor thermal comfort. Building and Environment, 46(4), 863-870. https://doi.org/10.1016/j.buildenv.2010.10.017

Instituto Nacional de Estadística y Censos de la República Argentina. (2010). Censo Nacional de Población, Hogares y Vivienda. https://www.indec.gob.ar/indec/web/Nivel4Tema-2-41-135

Jerez, S., \& Trigo, R. M. (2013). Time-scale and extent at which large-scale circulation modes determine the wind and solar potential in the Iberian Peninsula. Environmental Research Letters, 8(4), 044035. https://doi.org/10.1088/17489326/8/4/044035

Kamiya, M., \& Zhang, L.-Y. (2017). Finance for City Leaders Handbook. Improving Municipal Finance to Deliver Better Services. United Nations Human Settlements Programme (UN-Habitat).

Klinenberg, E. (2015). Heat Wave: A Social Autopsy of Disaster in Chicago. University of Chicago Press. https://books. google.com.ar/books?hl=es\&lr=\&id=LV6zBwAAQBAI\&oi=fnd\&pg=PR7\&ots=6ObMbYawSb\&sig=pPwe ZgWjukQyK2tyET8a5Nyw9Y\&redir_esc=y\#v=onepage\& $q \& \mathrm{f}=$ false

Knight, T., Price, S., Bowler, D., \& King, S. (2016). How effective is 'greening' of urban areas in reducing human exposure to ground-level ozone concentrations, UV exposure and the 'urban heat island effect'? A protocol to update a systematic review. Environmental Evidence, 5(3), 1-16. https://doi.org/10.1186/s13750-016-0054-y

Kurazumi, Y., Kondo, E., Ishii, J., Sakoi, T., Fukagawa, K., Bolashikov, Z. D., Tsuchikawa, T., Matsubara, N., \& Horikoshi, T. (2013). Effect of the Environmental Stimuli upon the Human Body in Winter Outdoor Thermal Environment. Journal of Environmental and Public Health, 2013, 418742. https://doi.org/10.1155/2013/418742 
Lazzari, S., Perini, K., \& Roccotiello, E. (2018). Green Streets for Pollutants Reduction. En G. Perez, \& K. Perini (Eds.), Nature Based Strategies for Urban and Building Sustainability (pp. 149-156). Elsevier. https://doi.org/10.1016/B978-0-12812150-4.00014-8

Lee, I., Voogt, J., \& Gillespie, T. (2018). Analysis and Comparison of Shading Strategies to Increase Human Thermal Comfort in Urban Areas. Atmosphere, 9(3), 91. https:// doi.org/10.3390/atmos9030091

Li, X., \& Ratti, C. (2018). Mapping the spatio-temporal distribution of solar radiation within street canyons of Boston using Google Street View panoramas and building height model. Landscape and Urban Planning, 191, 103387. https://doi.org/10.1016/J.LANDURBPLAN.2018.07.011

Lorda, M. A. (2008). Lógicas socioespaciales en el espacio periurbano de Bahía Blanca. Huellas, (12), 90-112. http://www.biblioteca.unlpam.edu.ar/pubpdf/huellas/ n12a06lorda.pdf

Lotufo Bueno-Bartholomei, C., \& Labaki, L. C. (2003). How much does the change of species of trees affect their solar radiation attenuation? International Conference on Urban Climate, 267-270. http://meteo.geo.uni.lodz. pl/icuc5/text/O_1_4.pdf

Matuszko, D. (2012). Influence of cloudiness on sunshine duration. International Journal of Climatology, 32(10), 1527-1536. https://doi.org/10.1002/joc.2370

Middel, A., Chhetri, N., \& Quay, R. (2015). Urban forestry and cool roofs: Assessment of heat mitigation strategies in Phoenix residential neighborhoods. Urban Forestry \& Urban Greening, 14(1), 178-186. https://doi.org/10.1016/J. UFUG.2014.09.010

Modificación Ordenanza 15523 de 2012 [Municipalidad de Bahía Blanca]. Arbolado Urbano y Espacios Verdes. 28 de noviembre de 2012. Pub. L. No. 16859.
Mohajeri, N., Gudmundsson, A., Kunckler, T., Upadhyay, G., Assouline, D., Kämpf, J. H., \& Scartezzini, J. L. (2019). A solar-based sustainable urban design: The effects of city-scale street-canyon geometry on solar access in Geneva, Switzerland. Applied Energy, 240, 173-190. https://doi.org/10.1016/j.apenergy.2019.02.014

Norton, B. A., Coutts, A. M., Livesley, S. J., Harris, R. J., Hunter, A. M., \& Williams, N. S. G. (2015). Planning for cooler cities: A framework to prioritise green infrastructure to mitigate high temperatures in urban landscapes. Landscape and Urban Planning, 134, 127-138. https:// doi.org/10.1016/j.landurbplan.2014.10.018

Oke, T. R., Mills, G., Christen, A., \& Voogt, J. A. (2017). Urban Climates. Cambridge University Press. https://doi. org/10.1017/9781139016476

Onishi, A., Cao, X., Ito, T., Shi, F., \& Imura, H. (2010). Evaluating the potential for urban heat-island mitigation by greening parking lots. Urban Forestry \& Urban Greening, 9(4), 323-332. https://doi.org/10.1016/j.ufug.2010.06.002

Patton, C. P., Alexander, C. S. y Kramer, F. L. (1978). Curso de Geografía Física. VICENS Universidad.

Perini, K., \& Roccotiello, E. (2018). Vertical Greening Systems for Pollutants Reduction. En G. Pérez, \& K. Periri (Eds.), Nature Based Strategies for Urban and Building Sustainability (pp. 131-140). Elsevier. https://doi.org/10.1016/B978-0-12$\underline{812150-4.00012-4}$

Pinassi, C. A. (2016). La configuración de un nuevo espacio turístico recreativo a través de la valorización del patrimonio cultural: el caso de Bahía Blanca. [Tesis de doctorado, Universidad Nacional del Sur]. Repositorio Institucional. http://repositoriodigital.uns.edu.ar/bitstream/123456789/3380/5/ Tesis PINASSI\%2C Carlos Andrés.pdf

Pozo-Vázquez, D., Tovar-Pescador, J., Gámiz-Fortis, S. R., Esteban-Parra, M. J., \& Castro-Díez, Y. (2004). NAO and solar radiation variability in the European North Atlantic region. Geophysical Research Letters, 31(5), 1-4. https:// doi.org/10.1029/2003GL018502 
Reyes, S. (2020). Forestación urbana para la adaptación al cambio climático (Documento para política pública). CEDEUS. https://www.cedeus.cl/forestacion-urbana-parala-adaptacion-al-cambio-climatico/

Rodríguez Algeciras, J. A. (2013). Morfología y microclima urbano [Tesis de Maestría, Universidad Politécnica de Cataluña]. Repositorio Institucional. https://wwwaie. webs.upc.edu/maema/wp-content/uploads/2016/07/TESINARodriguez-Jose-Abel.pdf

Rosake, P. y Ercolani, P. (2012). Los espacios de ocio de Bahía Blanca. Preferencias de la población en relación al uso de su tiempo libre. Realidad, Tendencias y Turismo, 10(1), 94115. https://dialnet.unirioja.es/servlet/articulo?codigo=7671075

Rowe, B. (2018). Green Roofs for Pollutants' Reduction. En G. Pérez, \& K. Periri (Eds.), Nature Based Strategies for Urban and Building Sustainability (pp. 141-148). Elsevier. https://doi.org/10.1016/B978-0-12-812150-4.00013-6

Sánchez, G., Serrano, A., \& Cancillo, M. L. (2012). Effect of cloudiness on solar global, solar diffuse and terrestrial downward radiation at Badajoz (Southwestern Spain). Óptica Pura y Aplicada, 45(1), 33-38. https://doi.org/10.7149/ OPA.45.1.33

Schroeder, R. y Garriz, E. (2009). ¿Es posible recuperar el espacio público? La ciudad de Bahía Blanca como caso de estudio. http://www.observatoriogeograficoamericalatina.org.mx/ egal12/Geografiasocioeconomica/Geografiaurbana/208.pdf

Scott, K. I., Simpson, J. R., \& Mcpherson, E. G. (1999). Effects of tree cover on parking lot microclimate and vehicle emissions. Journal of Arboriculture, 25(3), 129-142. https://pdfs.semanticscholar.org/ e034/06ba6437e4014d832273ee2dabfbe9332f42.pdf

Sengupta, M., Habte, A., Gueymard, C., Wilbert, S., \& Renné, D. (2017). Best Practices Handbook for the Collection and Use of Solar Resource Data for Solar Energy Applications (Second Edition). NREL.
Shahidan, M. F., Jones, P. J., Gwilliam, J., \& Salleh, E. (2012). An evaluation of outdoor and building environment cooling achieved through combination modification of trees with ground materials. Building and Environment, 58, 245-257. https://doi.org/10.1016/J.BUILDENV.2012.07.012

Shishegar, N. (2013). Street Design and Urban Microclimate: Analyzing the Effects of Street Geometry and Orientation on Airflowand Solar Access in Urban Canyons. Journal of Clean Energy Technologies, 1(1), 52-56. https://doi. org/10.7763/jocet.2013.v1.13

Tomson, T., \& Hansen, M. (2010). Fast changes of the solar irradiance. Estonian Journal of Engineering, 16(2), 176-183. https://doi.org/10.3176/eng.2010.2.06

Treberspurg, M. (2008). SolarCity: Linz Pichling. Springer. https://doi.org/https://doi.org/10.1007/978-3-211-69293-6

United Nations. (2015). World Urbanization Prospects (2014 Revision). https://esa.un.org/unpd/wup/publications/files/ wup2014-highlights.pdf

United Nations General Assembly. (2015). Transforming our world: The 2030 Agenda for Sustainable Development. https://sdgs.un.org/2030agenda

Urban, G., Migała, K., \& Pawliczek, P. (2017). Sunshine duration and its variability in the main ridge of the Karkonosze Mountains in relation to with atmospheric circulation. Theoretical and Applied Climatology, 131, 1173-1189. https://doi.org/10.1007/s00704-017-2035-7

Vásquez, A. E. (2016). Infraestructura verde, servicios ecosistémicos y sus aportes para enfrentar el cambio climático en ciudades: el caso del corredor ribereño del río Mapocho en Santiago de Chile. Revista de Geografía Norte Grande, (63), 63-86. http://dx.doi.org/10.4067/S071834022016000100005 
Weber, G. R. (1994). On the seasonal variation of local relationships between temperature, temperature range, sunshine and cloudiness. Theoretical and Applied Climatology, 50(1-2), 15-22. https://doi.org/10.1007/ BF00864898

World Meteorological Organization. (2017). Guide to Meteorological Instruments and Methods of Observation. Edición del autor.

Wong, N. H., \& Yu, C. (2005). Study of green areas and urban heat island in a tropical city. Habitat International, 29(3), 547-558. https://doi.org/10.1016/J.HABITATINT.2004.04.008

Zapperi, P. A. (2012). Hidrografía urbana de Bahía Blanca. Departamento de Geografía y Turismo, Universidad Nacional del Sur.

Zapperi, P., Ramos, M. B., Gil, V. y Campo, A. M. (2007). Caracterización de las precipitaciones estivales en el Suroeste bonaerense. Contribuciones Científicas GAEA, 19, 483-491. http://gaea.org.ar/contribuciones/ contribuciones_vol19_Zapperi-Ramos-Gil-Campo.pdf

Zhao, N., Zeng, X., \& Han, S. (2013). Solar radiation estimation using sunshine hour and air pollution index in China. Energy Conversion and Management, 76, 846-851. https:// doi.org/10.1016/J.ENCONMAN.2013.08.037 